\title{
Application of Medical Nursing Integrated Nursing Mode in Patients Undergoing Laparoscopic Roux-en-Y Gastric Bypass Bariatric Surgery
}

\author{
Li Zhang ${ }^{1}$, Shuqing Yu${ }^{2}$, Xiuli Wei ${ }^{2}$, Lina $\mathrm{Wu}^{2}$, Lilian $\mathrm{Gao}^{2}$, \\ ${ }^{1}$ Department of Comprehensive Ward 1, the First Affiliated Hospital of Jinan University, Guangzhou, China \\ ${ }^{2}$ Department of International Bariatric Center, the First Affiliated Hospital of Jinan University, Guangzhou, China \\ Email address: \\ zhanglijasmine@126.com (Li Zhang),1066267048@qq.com (Shuqing Yu),445827039@qq.com (Xiuli Wei), \\ 1479161420@qq.com (Lina Wu), 943309720@qq.com (Lilian Gao) \\ *Corresponding author
}

\section{To cite this article:}

Li Zhang, Shuqing Yu, Xiuli Wei, Lina Wu, Lilian Gao. Application of Medical Nursing Integrated Nursing Mode in Patients Undergoing Laparoscopic Roux-en-Y Gastric Bypass Bariatric Surgery. American Journal of Nursing Science. Vol. 9, No. 4, 2020 , pp. $215-219$.

doi: 10.11648/j.ajns.20200904.18

Received: May 14, 2020; Accepted: May 29, 2020; Published: June 8, 2020

\begin{abstract}
Purpose: To explore the effect of medical nursing integrated nursing mode on the quality of care of patients undergoing laparoscopic Roux-en-Y gastric bypass (LRYGB) bariatric surgery. Methods: A total of 60 patients from January 2018 to December 2018 were enrolled as the control group, and 60 patients from January 2019 to December 2019 were enrolled as the intervention group. In the control group, patients were cared by the routine nursing mode. In the intervention group, medical nursing integrated nursing mode was adopted in addition to the routine LRYGB nursing process. The waiting time of preoperative examination, preoperative HAMA and SDS scores, postoperative complication rates, time indicators of nursing quality and patient satisfaction in the two groups were collected and compared. Results: The waiting time of preoperative examination was shortened $(P<0.05)$, preoperative anxiety and depression were reduced $(P<0.05)$, the complication rates of postoperative bleeding, infection, wound pain and abdominal distension were reduced $(P<0.05)$, the time indicators of nursing quality were shortened $(P<0.05)$ and patient satisfaction was improved in the intervention group compared to the control group $(P<0.05)$. Conclusions: Application of medical nursing integrated nursing mode in patients undergoing LRYGB bariatric surgery can alleviate the negative emotions of anxiety and depression before surgery, reduce the postoperative complication rates, improve the quality of care and patient satisfaction.
\end{abstract}

Keywords: Medical Nursing Integrated, Bariatric Surgery, Satisfaction, Quality of Care

\section{Introduction}

Obesity refers to the accumulation and abnormal distribution of body fat caused by a variety of factors such as heredity, high-calorie, high-fat diet and lack of physical exercise [1]. The proportion of obese people has been increasing in recent years in China [2]. The treatment of obesity includes exercise, diet, medication and surgery. Bariatric surgery has a long-term and stable effect on weight loss and can treat or prevent obesity-related metabolic diseases, and laparoscopic Roux-en-Y gastric bypass (LRYGB) is one of the most effective bariatric surgical procedures [3]. Clinical studies have pointed out that obese patients may have anxiety, depression and other adverse emotions when facing surgery, and at the same time, due to the lack of bariatric surgery related knowledge, patients may have excessive anxiety, nervousness and other adverse emotions, which will have a serious impact on patients' physiology and psychology and affect the surgical efficacy and outcome [4]. Furthermore, the routine nursing mode in the perioperative period of bariatric surgery is mainly that nurses passively perform doctors' orders, and the lack of active communication with doctors, the neglect of patients' physiological and psychological changes and the lack of personalized nursing care for patients' postoperative diet and targeted nursing care to prevent patients' postoperative complications make it 
difficult to improve the quality of care of patients undergoing LRYGB bariatric surgery [5]. Therefore, in the nursing process, it is necessary to change the workflow, innovate the service mode, and promote collaboration between doctors and nurses to provide quality care to patients. Medical nursing integrated nursing mode is a process in which doctors and nurses provide medical care services to patients through open communication, coordination, joint decision-making and sharing of responsibilities under the premise of equality, autonomy, mutual respect and trust of professional knowledge and ability [6], which requires reasonable division of labor, close contact, information exchange and mutual cooperation between the two sides [7]. From 2019, our department of international bariatric center has started to pilot and implement the medical nursing integrated nursing mode in patients undergoing LRYGB bariatric surgery, which significantly improved the quality of care of the patients. The summary report is as follows.

\section{Data and Method}

\subsection{Research Objects}

In our hospital's international bariatric center, 60 patients admitted for LRYGB bariatric surgery from January 2018 to December 2018 were enrolled as the control group, and 60 patients admitted for LRYGB bariatric surgery from January 2019 to December 2019 were enrolled as the intervention group. In the control group, there were 20 females and 40 males with an average age of $33.47 \pm 10.88$ years old, an average body mass index (BMI) of $44.32 \pm 10.16 \mathrm{~kg} / \mathrm{m}^{2}$ and education levels: 1 case with primary school, 33 cases with middle school and 26 cases with university or above. In the intervention group, there were 19 females and 41 males with an average age of $35.12 \pm 10.90$ years old, an average BMI of $42.35 \pm 11.08 \mathrm{~kg} / \mathrm{m}^{2}$ and education levels: 1 case with primary school, 28 cases with middle school and 31 cases with university or above. There were no statistically significant differences between the two groups in general data $(P>0.05)$, suggesting comparability.

Inclusion criteria: (1) patients with a BMI greater than 40 $\mathrm{kg} / \mathrm{m}^{2}$ or a BMI between $28-40 \mathrm{~kg} / \mathrm{m}^{2}$ with more than 2 metabolic diseases, aged 16-68 years old; (2) patients who simply received elective LRYGB bariatric surgery for the first time; (3) patients who could correctly understand the relevant content of the scale and make answers. Exclusion criteria: (1) patients with the existence of cognitive or mental disorders; (2) patients who quitted the study; (3) patients who had comprehension and hearing impairment and could not complete the scale evaluation.

\subsection{Research Methods}

The control group patients received routine nursing mode, and the intervention group patients received medical nursing integrated nursing mode, specific as follows: (1) a medical nursing integrated bariatric surgical treatment team was established; the relevant medical nursing integrated cooperation system and process were formulated, and an integrated communication platform of doctors and nurses was established through a WeChat group; (2) after the patients were admitted to the hospital, the medical staff and the nursing staff made a joint ward round to assess the patients, reach an agreement on the nursing measures of the patients, and to build a medical nursing integrated record; (3) before the operation, the nurses participated in the preoperative discussion to understand the surgical methods of the patients, and jointly developed the preoperative care plans so as to decide when to place the gastric tube, when to clean the intestinal tract and to determine the amount of rehydration; (4) after the operation, both medical and nursing staff jointly developed treatment and nursing rehabilitation programs for the patients, and nurses also closely monitored the postoperative weight, diet, sleep, blood pressure and blood sugar of the patients.

\subsection{Evaluation Index}

In research, the waiting time of preoperative examination and time indicators of nursing quality were collected and compared. Patients' preoperative anxiety and depression were assessed using the Hamilton anxiety scale (HAMA) and self-rating depression scale (SDS) respectively. The scoring method of HAMA was as follows: 0 8 divided into no anxiety, 9 19 divided into mild anxiety, 20 34 divided into moderate anxiety, 35 points or more as severe anxiety [8]. SDS rating scale includes 20 rating items, according to the score: $0 \sim 34$ as no depression, 35 49 as mild depression, 50 69 as moderate depression, and 70 or more as severe depression [9]. The postoperative complication rates of bleeding, infection, wound pain and abdominal distension in the two groups were calculated. Patient satisfaction was assessed using a self-designed questionnaire, and the total score is 100 points, very satisfied meaning $\geq 80$ points, satisfied $\leq 79$ points and $\geq 60$ points, not satisfied $\leq 59$ points. Total satisfaction $=$ (very satisfied + satisfied)/total cases $\times 100 \%$.

\subsection{Statistical Methods}

Statistical analysis was performed using SPSS 19.0 software. The measurement data were expressed as mean \pm standard deviation $(\bar{x} \pm s)$ and were analyzed by $t$ test. The count data were analyzed by $\chi^{2}$ test. $P<0.05$ indicates that the difference is statistically significant.

\section{Result}

\subsection{Comparison of the Time Indicators Between the Control Group and Intervention Group}

By comparing the patient waiting time of preoperative examination and time indicators of nursing quality including time of reporting patient abnormal results to doctors and time of implementing doctors' orders accurately of the two groups, it was found all time indicators of the intervention group were significantly shorter than those of the control group $(P<0.05)$, as shown in table 1 . 
Table 1. Comparison of the time indicators between the two groups $(\overline{\boldsymbol{x}} \pm \mathrm{s})$.

\begin{tabular}{lllll}
\hline Group & Cases & $\begin{array}{l}\text { Waiting time of preoperative } \\
\text { examination (d) }\end{array}$ & $\begin{array}{l}\text { Time of reporting abnormal results } \\
\text { (min) }\end{array}$ & $\begin{array}{l}\text { Time of implementing orders } \\
\text { accurately (min) }\end{array}$ \\
\hline Control & 60 & $1.37 \pm 0.43$ & $7.46 \pm 0.26$ & $12.26 \pm 2.59$ \\
Intervention & 60 & $0.64 \pm 0.18$ & $3.59 \pm 0.35$ & $5.47 \pm 1.37$ \\
$t$ value & & 12.130 & 68.754 & 17.950 \\
$P$ value & & $<0.001$ & $<0.001$ & $<0.001$ \\
\hline
\end{tabular}

\subsection{Comparison of Patient Preoperative HAMA and SDS Scores Between the Control Group and Intervention Group}

Patient preoperative HAMA and SDS scores were assessed and the intervention group was found to have superior HAMA and SDS scores compared to the control group. The difference was statistically significant $(P<0.05)$, as shown in table 2 .

Table 2. Comparison of patient preoperative HAMA and SDS scores between the two groups $(\overline{\boldsymbol{x}} \pm \mathrm{s})$.

\begin{tabular}{llll}
\hline Group & Cases & HAMA & SDS \\
\hline Control & 60 & $13.42 \pm 2.73$ & $44.92 \pm 5.73$ \\
Intervention & 60 & $9.51 \pm 2.32$ & $36.25 \pm 6.48$ \\
$t$ value & & 8.454 & 7.764 \\
$P$ value & & $<0.001$ & $<0.001$ \\
\hline
\end{tabular}

\subsection{Comparison of Postoperative Complication Rates Between the Control Group and Intervention Group}

The postoperative complication rates of bleeding, infection, wound pain and abdominal distension in the intervention group were significantly lower than those of the control group $(P<0.05)$, as shown in table 3 .

Table 3. Comparison of postoperative complication rates of bleeding, infection, wound pain and abdominal distension between the two groups [n (\%)].

\begin{tabular}{|c|c|c|c|c|c|}
\hline Group & Cases & Bleeding & Infection & Wound pain & Abdominal distension \\
\hline Control & 60 & $8(13.33)$ & $6(10.00)$ & $12(20.00)$ & $11(18.33)$ \\
\hline Intervention & 60 & $1(1.67)$ & $0(0)$ & $4(6.67)$ & $3(5.00)$ \\
\hline$\chi^{2}$ value & & 4. 324 & 4.386 & 4.615 & 5.175 \\
\hline$P$ value & & 0.038 & 0.036 & 0.032 & 0.023 \\
\hline
\end{tabular}

\subsection{Comparison of Patient Satisfaction Between the Control Group and Intervention Group}

The total satisfaction of the intervention group (96.67\%) was significantly higher than that of the control group (85.00\%) $(P<0.05)$, as shown in table 4 .

Table 4. Comparison of patient satisfaction between the two groups [n (\%)].

\begin{tabular}{|c|c|c|c|c|c|}
\hline Group & Cases & Very Satisfied & Satisfied & Not satisfied & Total satisfaction \\
\hline Control & 60 & $31(51.67)$ & $20(33.33)$ & $9(15.00)$ & $51(85.00)$ \\
\hline Intervention & 60 & $45(75.00)$ & $13(21.67)$ & $2(3.33)$ & $58(96.67)$ \\
\hline$\chi^{2}$ value & & - & - & - & 4.904 \\
\hline$P$ value & & - & - & - & 0.027 \\
\hline
\end{tabular}

\section{Discussion}

LRYGB bariatric surgery is the most popular operation at present for treating patients with obesity and metabolic diseases [10]. Although LRYGB surgery can guarantee weight loss and has a lower perioperative mortality rate, patients with the higher BMI tend to have increased incidence of complications and increased surgical difficulty [11]. In addition, as one of the more common stressors in clinical practice, most patients will have different degrees of worry, fear and tension before surgery. Studies have proved that patients with negative emotions are prone to increase postoperative pain, which is not conducive to the early recovery of patients [12]. Therefore, to promote the prognosis of patients, the implementation of personalized perioperative nursing intervention that is specific to the characteristics of obesity is crucial to the success of surgery and postoperative recovery.

The results of medical nursing integrated nursing mode in this study showed that the time of reporting patient abnormal results to doctors and time of implementing doctors' orders accurately were shorter than those of the control group, confirming that through medical nursing integrated nursing mode could not only shift nurses from the passive performer to the critical thinker and the active practitioner who actively involved in the diagnosis and treatment of patients including the patient assessment, patient round, discharge plan and health education, but also ensure that the doctors' orders can be timely and accurately performed to the patients, because nurses can obtain the guidance of the doctors regarding nursing work and listen to the doctors' advice face to face 
through the medical nursing integrated nursing mode. The result of this study also showed that patients' waiting time of preoperative examination was shorter than that of the control group, indicating that the medical nursing integrated nursing mode could help to promote the efficiency of nursing work [13]. Furthermore, the results of this study showed that the preoperative anxiety and depression scores in the intervention group were lower than those of the control group, and the postoperative complication rates were also lower than those of the control group, thus confirming that through medical nursing integrated nursing mode could provide patient centered, personalized and targeted complication prevention perioperative nursing intervention for patients undergoing LRYGB bariatric surgery, so as to alleviate their preoperative anxiety and depression and reduce their postoperative complications such as bleeding, infection, wound pain, abdominal distension. Those findings are consistent with other studies [14]. In table 4, the satisfaction of the patients in the intervention group was higher than that of the control group. It can be seen that the patients' satisfaction with the nursing care was higher due to the fewer complications and faster recovery of the surgery under the medical nursing integrated nursing mode intervention [15]. However, the limitation of this study is that the sample size was small, and the differences between the indicators were only compared after intervention. In the future, the sample size should be enlarged, and family follow-up observation should be strengthened to further demonstrate the nursing effect of medical nursing integrated nursing mode.

\section{Conclusion}

We have adopted the medical nursing integrated nursing mode in patients undergoing LRYGB bariatric surgery and achieved positive results in nursing outcomes. We not only strengthened the communication and cooperation between doctors and nurses and promoted the medical nursing resources restructuring, but also alleviated the preoperative anxiety and depression, reduced postoperative complication rates and improved satisfaction and the quality of care of the patients in their perioperative period. Therefore, application of medical nursing integrated nursing mode in patients undergoing LRYGB bariatric surgery can provide efficient, safe and satisfactory nursing care for them, and the medical nursing integrated nursing mode is worth promoting and implementing.

\section{Ethics Approval and Consent to Participate}

Not applicable.

\section{Conflict of Interests}

The authors declare that they have no conflict of interests.

\section{References}

[1] Katharina, N., Stefan, K., \& Tobias, P. (2019). Diagnosis of obesity and use of obesity biomarkers in science and clinical medicine. Metabolism: Clinical and Experimental, 92 (3), 61-70.

[2] Zhang, L., Wang, Z., Wang, X., Chen, Z., Shao, L., \& Tian, Y. et al. (2019). Prevalence of abdominal obesity in China: results from a cross-sectional study of nearly half a million participants. Obesity, 27 (11), 1898-1905.

[3] Yousuf, G. S., \& Tim, S. (2019). Effectiveness of laparoscopic Roux-en-Y gastric bypass and sleeve gastrectomy for morbid obesity in achieving weight loss outcomes. International Journal of Surgery (London, England), 70, 35-43.

[4] Lai, C., Aceto, P., Petrucci, I., Castelnuovo, G., Callari, C., \& Giustacchini, P. et al. (2019). The influence of preoperative psychological factors on weight loss after bariatric surgery: A preliminary report. Journal of Health Psychology, 24 (4), 518-525.

[5] Christopher, D. R., Stacy, B. A., Chao, T., Anthony, P. T., John, M. M., \& Philip, S. R. et al. (2018). Which postoperative complications matter most after bariatric surgery? Prioritizing quality improvement efforts to improve national outcomes. Surgery for Obesity and Related Diseases, 14 (5), 652-657.

[6] Chen, J., Zhang, Z. X., \& Xie, H. (2011). Research progress of medical cooperation. Chinese Journal of Nursing, 46 (11), 1136-1139.

[7] Cypress, B. S. (2011). Exploring the concept of nurse-physician communication within the context of health care outcomes using the evolutionary method of concept analysis. Dimens Crit Care Nurs, 30 (1), 28-38.

[8] Qiang, H., Wang, L. L., Zhou, J. S., \& Sun, M. (2013). Surgical cooperation in laparoscopic adjustable gastric band volume reduction surgery. Journal of Shanghai Nursing, 13 (5), 58-60.

[9] Shao, Q., He, J. H., \& Wang, J. Q. (2014). Nursing experience of laparoscopic sleeve gastrectomy for adolescent moropathic obesity. Nursing \& Rehabilitation, 13 (10), 954-956.

[10] Chopra, A., Chao, E., \& Etkin, Y. et al. (2012). Laparoscopic sleeve gastrectomy for obesity: Can it be considered a definitive procedure? Surgical Endoscopy, 26 (3), 831-837.

[11] Nasser, H., Ivanics, T., Leonard-Murali, S., Shakaroun, D., \& Genaw, J. (2019). Perioperative outcomes of laparoscopic Roux-en-Y gastric bypass and sleeve gastrectomy in super-obese and super-super-obese patients: A national database analysis. Surgery for Obesity and Related Diseases, 15 (10), 1696-1703.

[12] Baird, A., \& Sheffield, D. (2016). The relationship between pain beliefs and physical and mental health outcome measures in chronic low back pain: Direct and indirect effects. Healthcare, 4 (3), 58.

[13] Cui, J. J. (2020). Application of integrated medical care model in orthopedic nursing. Journal of Psychology, 15 (06), 99.

[14] Zhang, A. N., \& Li, W. H. (2019). Application effect of rapid rehabilitation model integrating medical care and health care in perioperative nursing. Clinical Medical Research and Practice, 4 (12), 157-158. 

Roux-en-Y Gastric Bypass Bariatric Surgery

[15] Deng, R. Y. (2020). Observation on the effect of medical care integration model on the intervention of adverse emotions and risk events in patients in the comprehensive ward of geriatrics department. Chinese and Foreign Medical Research, 18 (10), 153-155. 